\title{
Decision Making About Rural Physiotherapy Service Provision Varies With Sector, Size and Rurality
}

\author{
Robyn Adams \\ James Cook University, robyn.adams@jcu.edu.au \\ Anne Jones \\ James Cook University \\ Sophie Lefmann \\ University of South Australia \\ Lorraine Sheppard \\ University of South Australia
}

Follow this and additional works at: https://nsuworks.nova.edu/ijahsp

Part of the Physical Therapy Commons, and the Physiotherapy Commons

\section{Recommended Citation}

Adams R, Jones A, Lefmann S, Sheppard L. Decision Making About Rural Physiotherapy Service Provision Varies With Sector, Size and Rurality. The Internet Journal of Allied Health Sciences and Practice. 2015 Apr 01;13(2), Article 7.

This Manuscript is brought to you for free and open access by the College of Health Care Sciences at NSUWorks. It has been accepted for inclusion in Internet Journal of Allied Health Sciences and Practice by an authorized editor of NSUWorks. For more information, please contact nsuworks@nova.edu. 


\title{
Decision Making About Rural Physiotherapy Service Provision Varies With Sector, Size and Rurality
}

\begin{abstract}
Introduction: Decisions about physiotherapy service provision occur within the context of organisations, locations, and settings. The uniqueness of rural communities means it is important to consider contextual factors when making decisions about rural health services. As literature describing decision making about rural physiotherapy services is limited, this study sought perspectives on service level decision making (SLDM) from a range of stakeholders. The research approach needed to support consideration of both location and the broader health system. Method: A sequential mixed methods approach within a systems theory-case study heuristic provided the framework to explore rural physiotherapy SLDM. The investigation site, a large area of one Australian state, contained a mix of regional, rural, and remote communities. Perspectives on SLDM were obtained through surveys of physiotherapists, colleagues, and managers, with follow up interviews of a purposeful sample of participants. Results: Responses from physiotherapists, colleagues, manager surveys $(n=34)$, and in-depth interviews $(n=19)$ revealed commonalities and differences between sites and sectors. Available skill and expertise were common considerations across sites. Decisions about prioritisation of services occurred in both public and private settings; however, organisational priorities were a greater influence in the public sector and financial viability in the private sector. Service size influenced the perceived degree of autonomy of physiotherapists in SLDM, with physiotherapists in smaller sites having more independence. Directions from health facility management and the increasing need to prioritise services were reflected in physiotherapy responses from larger facilities. National health reforms and state level priorities were noted as influencing factors, as were connection to community, rurality, and the distance from decision makers. Conclusion: This study provides insight into decisions informing rural physiotherapy service provision. Understanding context and diversity is important to understanding local health service decisions. System level influences from macro and meso level decision makers provide the framework within which micro level physiotherapy SLDM occurs. Service sector, size and rurality then further qualify local service options and influence SLDM. Decisions about service provision need to take into consideration the current availability of services, the context of each location and skill mix required.
\end{abstract}




\title{
TIUAHSP
}

\section{The Internet Joưnal of Allied Health Sciences and Practice}

Dedicated to allied health professional practice and education

Vol. 13 No. 2 ISSN 1540-580X

\section{Decision Making About Rural Physiotherapy Service Provision Varies With Sector, Size and Rurality}

\author{
Robyn Adams, BAppSc (Phty), PhD1 \\ Anne Jones, BSc, MPhty, PhD² \\ Sophie Lefmann, Physiotherapy (Honours), $\mathrm{PhD}^{3}$ \\ Lorraine Sheppard, BAppSc (Physiotherapy), MBA, PhD ${ }^{4}$
}

1. Adjunct Lecturer, Discipline of Physiotherapy, James Cook University, Townsville, Queensland.

2. Senior Lecturer, Discipline of Physiotherapy, James Cook University, Townsville, Queensland.

3. Lecturer, Discipline of Physiotherapy, University of South Australia, Adelaide, South Australia.

4. Professor, Discipline of Physiotherapy, James Cook University, Townsville and School of Health Sciences, University of South Australia, Adelaide, South Australia.

Australia

\begin{abstract}
Introduction: Decisions about physiotherapy service provision occur within the context of organisations, locations, and settings. The uniqueness of rural communities means it is important to consider contextual factors when making decisions about rural health services. As literature describing decision making about rural physiotherapy services is limited, this study sought perspectives on service level decision making (SLDM) from a range of stakeholders. The research approach needed to support consideration of both location and the broader health system. Method: A sequential mixed methods approach within a systems theory-case study heuristic provided the framework to explore rural physiotherapy SLDM. The investigation site, a large area of one Australian state, contained a mix of regional, rural, and remote communities. Perspectives on SLDM were obtained through surveys of physiotherapists, colleagues, and managers, with follow up interviews of a purposeful sample of participants. Results: Responses from physiotherapists, colleagues, manager surveys $(n=34)$, and in-depth interviews $(n=19)$ revealed commonalities and differences between sites and sectors. Available skill and expertise were common considerations across sites. Decisions about prioritisation of services occurred in both public and private settings; however, organisational priorities were a greater influence in the public sector and financial viability in the private sector. Service size influenced the perceived degree of autonomy of physiotherapists in SLDM, with physiotherapists in smaller sites having more independence. Directions from health facility management and the increasing need to prioritise services were reflected in physiotherapy responses from larger facilities. National health reforms and state level priorities were noted as influencing factors, as were connection to community, rurality, and the distance from decision makers. Conclusion: This study provides insight into decisions informing rural physiotherapy service provision. Understanding context and diversity is important to understanding local health service decisions. System level influences from macro and meso level decision makers provide the framework within which micro level physiotherapy SLDM occurs. Service sector, size and rurality then further qualify local service options and influence SLDM. Decisions about service provision need to take into consideration the current availability of services, the context of each location and skill mix required.
\end{abstract}

\section{INTRODUCTION}

Decisions about physiotherapy service provision occur within the context of organisations, locations, and settings. Understanding the varying contexts can provide insight into decision making about service provision. Context is especially important in rural and regional areas. The self-evident phrase,"If you have seen one country town, you have seen only one country town" is indicative of the uniqueness of rural communities. ${ }^{1}$ This uniqueness of place then combines with other factors to provide the context in which health services, such as physiotherapy, are provided. Other influencing factors include location and availability of other 
services, internal factors such as organisational imperatives, culture, and workforce capacity and capability, and external factors including health financing, policy, and workforce supply.

Physiotherapy services in Australia are provided through the public and private sectors of the health system. ${ }^{2,3}$ Public services are primarily funded and provided through state and territory health departments, with the Australian Government funding programs to improve access to services in underserved areas such as rural and remote communities. ${ }^{4-6}$ Private services are funded primarily by a fee-for-service model, with an increasing range of rebates or fee supports available through private health insurance, third party insurance, Work Cover, and Medicare. 7,8

Inpatient, outpatient, and community physiotherapy services are provided in the majority of publicly funded hospitals, with specialist physiotherapy services such as intensive care, burns, rheumatology, orthopaedics, geriatrics, paediatrics, and rehabilitation established in metropolitan and larger regional facilities. Musculoskeletal physiotherapy continues to form the core of private sector physiotherapy services. ${ }^{2}$ Smaller rural settings require generalist physiotherapists as they must demonstrate high level of skills in a variety of areas of physiotherapy. ${ }^{3,9}$ Articles describing the work practices of physiotherapists in regional, rural, and remote areas reveal differences in service provision between locations reflecting a responsiveness to context, setting, and location. 2,3

Decisions are made at all levels of a system or organisation. Decision making within health services occurs at micro, meso, and macro levels, which include decisions at the clinical or service level, the regional or facility level, and the state and national level respectively. ${ }^{10,11}$ At a macro level, health policies frame the broad context for health service provision. Meso-level decisions use numerous approaches and are considerably influenced by macro-level decisions. ${ }^{12}$ Two types of decision making occur at the micro level: clinical decisions at the patient-therapist interface, and individual decisions about service provision.

Clinical decision making at the patient-therapist level is well described, but descriptions of decision making at the service level are not readily evident in the physiotherapy literature. ${ }^{13-21}$ There is an emerging literature describing physiotherapy service provision in regional, rural, and remote areas, but little beyond caseload prioritisation describing service level decision making (SLDM).2,3,22 Differences in services provided and work settings between private and public physiotherapy services suggest different SLDM processes may be adopted. Similarly, the size of the service or facility and the geographical location in which the services are provided potentially influence SLDM. How and to what extent these factors influence physiotherapy SLDM in the varying rural settings is not evident in the literature.

This study sought participant perspectives on decision making about the provision of physiotherapy services in rural communities within the investigation area. Considering stakeholder perspectives within a systems framework across multiple locations enabled the researchers to discern differences between sector, size, and rurality. Obtaining perspectives of a range of stakeholders, such as multidisciplinary team member colleagues, and facility, service, or regional managers, provides a richer view of SLDM than a physiotherapy only perspective would provide. Descriptions of health service provision within the context of systems and rural location or place are important as the broader health system can both constrain and enable local health responses..$^{23}$ Decision making about rural physiotherapy service provision was explored within a systems-theory case study heuristic, which enabled consideration of both location and broader health system impacts.

\section{METHOD}

A sequential mixed methods approach within a systems theory-case study heuristic was used to identify and explore factors affecting rural physiotherapy service provision and related service level decision-making. A preliminary quantitative component preceded and guided the main qualitative data collection by informing purposive sampling and establishing preliminary results for further in-depth exploration. This research approach supported consideration of the impact of contextual factors such as service location, size or sector.

Stakeholder perspectives were obtained through surveys and in depth interviews. The investigation site was a large area of one Australian state with a mix of regional, rural, and remote communities. Rather than a formal geographic classification of location, the researchers were interested in the participants' perspective of rurality (PPR). Participants were asked to describe their community as regional, rural, or remote. PPR was then combined with the number of full time equivalent (FTE) public sector physiotherapists to form a matrix to inform case selection. Six case types in which to explore rural physiotherapy SLDM were identified within the study area: for example, a rural community with a sole physiotherapist (Rural $\leq 1$ ) or regional centre with more than 10 FTE physiotherapists (Regional $>10$ ). The six case types that emerged were Rural $\leq 1$, Rural 2-3, Rural 4-10, Rural-Remote 4-10, Regional 4-10, and Regional $>10$. Where common issues were identified, facilities are grouped as small (Rural $\leq 1$, Rural 2-3) or large (Rural 4-10, Rural-Remote 4-10; Regional 4-10; and Regional >10).

(c) The Internet Journal of Allied Health Sciences and Practice, 2015 
Purposefully designed surveys for physiotherapists, colleagues, and managers had been piloted in another rural location. The physiotherapist survey was sent to public sector physiotherapists in the investigation area. These physiotherapists were also asked to identify stakeholders involved in decision making about physiotherapy service delivery, including other public sector physiotherapists, physiotherapists in private practice, colleagues, and managers, and to distribute stakeholder-specific surveys. Physiotherapist participants were invited to participate in a follow-up interviews. Semi-structured, in-depth interviews were then conducted with a purposive sample of physiotherapists and other decision makers to gain more information on factors that influence decision making.

Ethics approval was obtained from a local human research ethics committee and from James Cook University (H3799). Site specific approval was obtained for each location in the study. Information sheets were provided to all potential participants. Written consent was obtained from all survey and interview participants. Data collection was undertaken from January to September 2012.

Survey data were analysed using descriptive statistics and recorded in Microsoft Excel spread sheets. Thematic analysis of open ended survey questions informed the development of initial themes and areas for further exploration in interviews. Interviews were audiotaped with full interview transcripts and a summary developed by the researcher provided to interview participants for their review and comment. Recorded interviews were transcribed verbatim and entered into NVivo version 10. An iterative approach was used to guide the thematic analysis of qualitative data. Thematic analysis developed themes and concepts which were coded and then used to frame and account for the data. The principal researcher completed the initial analysis with coresearchers double coding one third of the interviews to add to the depth of analysis.

\section{RESULTS}

Survey responses from 34 participants (21 physiotherapists and 13 colleagues/managers) provided an initial picture of rural physiotherapy SLDM. In addition to the many common issues identified by physiotherapists and their colleagues or managers (Table1), physiotherapists indicated that potential outcomes of the intervention and the impact of students and the media were influences on SLDM. Colleague and manager survey respondents highlighted historical influences and staffing to population ratio, and one participant noted they had no input into decision making about physiotherapy services. Follow up in-depth interviews (14 physiotherapist, and 5 colleagues/managers) enabled further exploration of SLDM. Survey participants were coded alpha-numerically in the following groups: public sector physiotherapists (P), private sector physiotherapist (PP), and colleagues and managers (CM). Interview participants have been coded alpha-numerically in the following groups: public physiotherapists (A), private physiotherapists (B), colleagues (C), and managers or other decision makers (D).

\section{Survey Data Suggest Commonalities and Differences between Sites and Sectors}

In response to the question "What factors do you consider when making decisions about which physiotherapy service to provide within your facility/service?" commonalities and differences emerged between sites and sectors. Physiotherapists took a range of factors into consideration when making decisions about service provision. Available skill and expertise were common considerations across public and private, and small and large facilities, as were patient or community needs (Table 1). Common to participant responses in both small and large public sites were considerations of evidence and potential outcomes of providing an intervention, staffing levels, and availability of other services. While decisions about service priorities occurred in both public and private settings, the underpinning factors differed. Acuteness of inpatients was a common public sector consideration, whereas time was a key consideration in private practice. Distinguishing factors between these two sectors were the impact of organisational priorities (prioritised by the public sector) and financial viability (prioritised by the private sector).

A difference between larger regional and smaller rural sites emerged from the physiotherapists' responses about how decisions were made about which physiotherapy services were provided. Responses such as "physiotherapist has 90\% control" [P15] and "I make them" [P14], from smaller sites suggest a degree of independence in SLDM. Physiotherapy department managers in larger services had decision making responsibility; however, it was constrained by the priorities of the health facility and by directions received from facility management. State level priorities were also noted as influencing what services were funded, particularly by physiotherapists in larger facilities.

Private practice physiotherapy respondents indicated the practice principal made the decisions about service provision. Consultation with staff and consideration of their areas of interest were common in larger practices. Other considerations were confidence to provide specific services, service gaps and profitability.

(C) The Internet Journal of Allied Health Sciences and Practice, 2015 
Table 1. Factors Influencing Rural Physiotherapy SLDM Identified In Surveys

\begin{tabular}{|l|l|}
\hline Physiotherapists & Colleagues/Managers \\
\hline Outcome and potential impact of service or intervention & Skill level \\
Available skills & Skill mix \\
Availability of staff & Expertise \\
Staffing level & Staffing ratio to population \\
Resources and costs & Staffing levels \\
Patient or community need & Vacancies \\
Priorities-department, facility and network & Workload \\
Availability other services & Institutional demands \\
Students & Agreed core business \\
Confident and competent staff & Budget pressures \\
Community and referrer expectations and referrals & Increased services without an increase in physiotherapists \\
Organisational plans and directives & Needs of community \\
Hospital business & Common health problems \\
Limits & Availability of other services locally and at discharge destination \\
\hline
\end{tabular}

\section{Exploration of Factors Influencing Physiotherapy Service Level Decision Making}

In-depth interviews with purposefully sampled physiotherapists and other key decision makers enabled further exploration of rural physiotherapy SLDM. Consistent with themes within the survey data, a number of common factors quickly emerged as interviews were conducted (see Table 2). These factors were explored, refined, or explained further by successive interview participants. The factors reveal the impact of broader system decision making on the provision of physiotherapy services. Exploring SLDM across rural sites provided insight into both similarities and differences, and the interconnected characteristics of systems.

Table 2. Factors Influencing Rural Physiotherapy SLDM Explored In Interviews
\begin{tabular}{|l|l|}
\hline Health Reforms & National, State and Local Health Reforms \\
\hline Health financing & $\begin{array}{l}\text { Activity based funding (ABF) } \\
\text { austerity measures }\end{array}$ \\
\hline Workforce & $\begin{array}{l}\text { Capacity; skill and expertise; "stayers and leavers"; access to continuing professional } \\
\text { development (CPD) }\end{array}$ \\
\hline Decision drivers & $\begin{array}{l}\text { Money, organisational priorities; new funding priorities, and market capacity to sustain } \\
\text { private practices; financial viability: evidence and data }\end{array}$ \\
\hline $\begin{array}{l}\text { Distance from regional, state and } \\
\text { national decision makers }\end{array}$ & Knowledge of local issues; voice at the table \\
\hline Connection to community & Visibility; accountability \\
\hline Rurality and size & Recruitment and retention; generalist skill set; isolated; paperwork \\
\hline
\end{tabular}

${ }^{*}$ Activity Based Funding (ABF) is a way of funding hospitals whereby they get paid for the number and mix of patients they treat.

\section{Health Reforms}

Health reforms initiated at a national level were a stimulus for change at state, regional, and facility levels. Interview participant comments reflected on the influence of the 2011 National Health Reform Agreement and subsequent state level decisions on organisational structures, governance, and allocation of funding. The establishment of local health districts, each a corporate body governed by a local health service board, was a state level organisational and administrative change that featured in participant comments. Comments about the local impact of macro level changes revealed a sense of frustration and a perceived disconnect of decision makers from the daily challenges of service delivery. Participants discussed the reforms in terms of constant administrative change, increased organisational risk emerging from changes to health financing, and constraints on service delivery.

D4: I'll start with the state reforms ... there's two very clear pieces of reform that came through. One was January 2011, when the districts were established ... then the election in March I think was probably the biggest change ... the boards came back.

D4: In terms of the federal reform the risk for us at the moment is that we're too small to really operate under ABF 
A9: Look it's this constant change of administration - the changes to the health system itself and the change of government ... they change things all around; change health districts - they seem to bring a structure in ... and they still put different people on it in these admin jobs, ... you still sort of feel you're still top heavy and they always said that we would get more on the ground and we never did.

\section{Health Financing}

Health system financing, notably the move to activity based funding (ABF), fiscal austerity measures, and new targeted funding emerged as key influencers of SLDM. The introduction of ABF impacted on physiotherapy SLDM in both large and small sites of this study. Decisions made to optimise revenue within the ABF model created organisational priorities and influenced subsequent decisions about management of specific patient groups. This necessitated physiotherapy service re-prioritisation, which for some physiotherapists, generated a level of frustration, while others were more pragmatic.

A3: I just don't understand why they would choose to pick orthopaedic in-patients, [which] seems to be their area of focus at the moment for ABF. What can we do to make the most money out of these people - they're the most predictable. I can tell them exactly how long they're going to stay for ... we've got medical patients who will stay for months and months and months because no one will make a decision about the medical care ... You've got patients there with no plans, no decisions, so why are they focussing on predictable patients to try and shave time off, it makes no sense whatsoever.

Managing length of stay (LOS), a key strategy to manage patient flow and to optimise funding within ABF facilities, had flow on effects to smaller rural "block-funded" health services. Decreasing LOS of patients in larger regional facilities was achieved, where possible, by discharging patients earlier or transferring patients to nearby rural hospitals. Access to outpatient physiotherapy services following discharge varied depending on location and service capacity, while the transfer of patients changed the inpatient activity and acuity of rural hospitals. Such changes require consideration in local decision making about provision of physiotherapy services in order to meet shifting priorities.

A1: Yes acuity and being in hospital and getting people out of hospital ... because anybody in health that is looking at dollars looks at length of stay, and it's the only thing that counts.

A8: That's economics, the [nearby] Base [hospital] just send them anywhere they can to get them out ... I mean when I told them that I will be away they just said "Oh we'll still send them there,"

Tightening of state budgets and changes to national health financing combined to influence regional and facility decision making. Fiscal austerity measures were implemented to meet regional and facility financial targets specified in the purchasing and performance agreements with state health authorities. Strategies adopted included bed closures and reduction in FTE staffing levels. Where physiotherapy FTE reductions occurred, the resultant decreased capacity necessitated decisions about service priorities, including decisions about services that would not be provided. As a result, there were service reductions and gaps in areas such as rehabilitation, outpatient services, and paediatric services, particularly for children with developmental delay or disability. Private physiotherapists also noted these gaps, but did not consider service provision to these client groups to be within their service scope.

A1: The staffing establishment that we have now is significantly reduced on what we had had for many years before hand. 2008-9-10 was a period of considerable external review for this whole area ... a consistent figure of around $25 \%$ of allied health positions [were cut] by some mechanism or another. That severely curtailed our ability to provide outpatient services. We've had to basically can [cease] any out-patient rehabilitation service so when people have gone home after rehab, [we have] very little ability to monitor programs or run a basic sort of weekly exercise class for those teetering on the edge of keeping them fit and functioning in their home versus losing ability and then needing more care.

B1: I've been here for 32 years, I'm in private practice, I do general physio, and over the years l've probably tailored it to the things I like doing, so I don't do much neurological, I don't do many kids and I don't do anything really long term, chronic. I keep it to what I'm happy with. I believe everybody's got a right to good health care but that doesn't necessarily fit in to the private practice model.

Both opportunities and challenges emerged from funding enhancements for specific services, such as subacute care (eg rehabilitation), post-acute care, and emergency department physiotherapy. Wariness and frustration accompanied new funding

(c) The Internet Journal of Allied Health Sciences and Practice, 2015 
in facilities recently affected by reductions in staff or where new funding established specific service teams or positions. Where physiotherapy service capacity had been reduced by staff cuts, the addition of new program funding at times "clawed back" capacity to pre-cut levels, but with less flexibility. Perhaps in response to decreases in existing services, physiotherapists in the newly funded services were approached to provide services beyond the specified service criteria, generating a level of frustration or angst. Further consequences were fragmentation of services and decreased flexibility in physiotherapy service provision, particularly where new funding was used to establish separate service teams or positions. When considered together, participant comments reflect missed opportunities to collaborate on decision making about physiotherapy service provision.

A1: Sub-acute [patient care funding] was an extra pot of money that was given out by the Commonwealth [national] government to enhance sub-acute rehab, stroke, psychogeriatric, and all that sort of stuff ... and those pots of money and those services really only just redistributed stuff that originally happened.

D1: I think the pots of money are probably a big challenge, I find at the moment, it's a challenge in terms of how we work as teams with different pots of money and making sure that there's that cohesion.

A2:.Each day, you'll be rung up and you'll field referrals where [they say] we've tried here, we've tried there, we've tried everywhere else, do you think you could fit them in, would you mind? And quite frankly, a bean counter in Canberra can absolutely draw a line there, but me as someone who lives and works in the community, l'm much more uncomfortable with drawing the line that says you are eligible and you are not due to geography or the year you were born or whatever. It's a limited pot of money but the need is there.

A3: ED [physio] is one of our artificial priorities because realistically, it wouldn't come up as a first thing that we would do, but because of the funding, it's one that's going to be maintained regardless of what is happening anywhere else.

Funding that enables access to private physiotherapy services was an important but not a key determining factor in SLDM by private physiotherapists in this study. There was a view expressed by a number of private physiotherapists that private practitioners provided services to "the private community" defined by one participant as "those wishing to utilise Private Health [insurance]" [B5]. The percentage of private practice clients utilising private health insurance or where physiotherapy was otherwise funded varied between practices.

\section{B3: It would have to be over 50\%, l've never really looked at it. Actually it might be about 50\%}

B4: It's probably 25\% workers comp, probably 25\% DVA [Department of Veteran Affairs] funding, probably $40 \%$ private, and about $10 \%$ Medicare ... and the Medicare ones, you know, they're as poor as poor, they just can't afford it

B5: We do Workcover, we do DVA, and we do the Medicare program so the Enhance Primary Care program and we do Compulsory Third Party

\section{Workforce Key to Service Sustainability}

The capacity to deliver and sustain a service emerged as a critical factor informing decisions about service provision. Workforce was a key factor in service sustainability with available expertise then shaping what services could be offered. Staffing levels, skills, and expertise were listed as key workforce factors across sites and sectors. Expertise was a significant influencing factor in both public and private services when physiotherapist made decisions to provide or not provide a service. Private physiotherapy providers indicated they only provided services for which they were skilled. When public sector physiotherapy services were unable to obtain the required expertise, gaps in local services occurred. For example, filling specialist paediatric roles was especially challenging as this is regarded as a highly specialised area with small numbers of "experts" available.

\section{B5: I think staffing is the biggest issue here}

A3: We could have a lovely "see everyone" sort of service but we don't have the staffing, and we also don't have the expertise to do it. We're very wary of opening up services that we can't sustain.

A1: Yes, you're looking for a more specialist skill set, that's more challenging to start off with ... and rural ... and if it's part time, then it's whether you start striking at luck it lucky because they're here for another reason and that just reduces your odds hugely. 
A1: Then there's kids that go into nowhere land. When [the specialist paediatric physiotherapist] leaves here, if there is no-one with a reasonable skill level, there will be a gap because I can't pick up that ... I can't do it.

Access to professional development and education was an important workforce factor noted across sites and sectors. The ability to access education helped inform physiotherapists' decisions to move to or stay in rural areas. Improved retention supports service sustainability and workforce stability enables a more proactive or planned approach to SLDM. Enhanced access through webinars and podcasts, while viewed positively, were not as valued as face to face education and workshops.

A5: ... the only thing I was going to add to it was professional development - it's a big difference [between city and country] - you have to go searching and over time l've developed a really good little network

B4: Education's really important, whether I stayed and what I did, and what I learnt, [Q: So that was one of your deciding factors about staying?] Critical factor about whether I wanted to stay as a physio ... I wasn't sure if I could leave the city and live in the country.

\section{Key Decision Drivers Vary Across Sites}

Key decision drivers varied with facility size, funding models, and function, with funding key to many decisions. Decision drivers included money, organisational priorities, new funding priorities, market capacity to sustain private practices, and perception and knowledge of the role of physiotherapy. Regional hospital physiotherapy service decisions were framed within the business of the hospital. In larger ABF funded facilities, organisation priorities of LOS, bed block, waiting lists and the "bottom line" were common in physiotherapy participant responses.

A3: Yes, I think waiting lists, especially for orthopaedics, waiting lists will drive decisions.

D1: [l] just think it's about being sucked into acuity. It's about getting people out of hospital ... and it's about the whole hospital, the business of the hospital.

Newly funded services, with defined service criteria and targeted patient populations and reporting requirements, were seen to create service silos and decrease flexibility. Service criteria included age, condition, and geography, for example "over 65 orthopaedic" [A5] or "within $20 \mathrm{~km}$ radius" [D1]. The funding and reporting requirements of new services established new priorities for service providers, and while enhancing services in some areas, created inequities and challenges due to the varied implementation.

A1: Sub-acute [program funding] was supposed to be for the whole region. But it was very good at giving gold standard service to those few ortho-geris that were in the ward and then encouraging them to be taken, transferred out to peripheral hospital. It happened a lot from [the nearby regional centre] and going out; we saw very little effect.

D1: ... so when you're all funded from little pots of money it can make you more silo and insular.

A5: ... sub-acute is a great concept of getting the communication and the responsibility that will follow the patient [over 65 orthopaedic] and follow them all the way ... and it's worked pretty well for us.

D1: Especially when you've got to report against that funding, against that criteria, it limits the amount of people that are willing to be flexible around the edges. That's a risk with the pots of money and something we're going to have to overcome otherwise it's going to be problem with Australian healthcare.

Different perspectives on issues that influence decisions emerged from the stakeholder groups depending on organisational position, distance from regional, state, and national decision makers, or connectedness to the community. One example is the different perspectives about centralisation of physiotherapy services and replacing locally based services with specialist outreach or consultant models. A regionally based decision maker espoused these models in contrast to physiotherapists based in smaller rural communities who advocated continuation of locally based physiotherapists who can build and maintain knowledge and relationships and be responsive to local community need. Either decision can be justified. The perspectives also reflect the organisational level of the stakeholder, their organisational role, and focus. The following participant comments reveal differing perspectives and motivations on just one issue (centralised or locally based physiotherapy services). The first perspective being of organisational imperatives such as efficiency and the second, the value added to communities by embedded services.

(c) The Internet Journal of Allied Health Sciences and Practice, 2015 
D2: ... [earlier I] talked about the fact that the roles as they are probably won't continue, that we need to rethink how the Allied Health assistants and maybe it's a consultative service to some of those communities that were historically always occupied by a physiotherapist. We still have some physiotherapists working in towns of less than 500 people, working full time equivalent roles. Now as an organisation that isn't the most efficient way of running a service, so we will look at new models of care

A7: ... what I notice in communities is the more services that are in a smaller community, the richer that community actually is. There is a certain wealth to the community - a diversity within the community

Community need, local demand, and practitioner defined priorities were more common decision drivers in smaller rural facilities than in the larger regional facilities. Physiotherapist responses in smaller rural health services suggested they had the scope to decide which services to provide. Smaller services appeared to prioritise outpatient services in contrast to larger regional facilities where inpatient activity dominated service priorities.

A8: So it's all open ... but at the end of the day I choose to run four hours of my twenty hours for exercise class, that's been never any problem.

A5: ... but in terms of prioritising - if l've got someone with pneumonia being referred for a chest infection - I would prioritise a paediatric child who has a serial cast following botox, so they are outpatient, but I would still prioritise [them] over an inpatient with pneumonia [who] is on antibiotics [where] I am going to have a limited effect with that patient.

A7: I would try and always aim for the exercise classes to keep running [and] triage your outpatients that certain chronic conditions will have to simply wait with some compassion.

Changes in health financing and increased accountabilities for delivering services within budget perhaps explain one common participant perception that "dollars drive decisions." This included decisions about service delivery, staffing levels, and service continuity.

A3: Absolutely, ridiculous and I just don't understand, no thought about outcomes, no thought about patients, it's just about revenue, which I just think is backwards.

A2: I think there is a real sense that suddenly someone will draw the line and go we can't afford this or this isn't generating enough money and so we will cut this service. There is certainly a sense that you are the one dangling. I think that there is a commitment; that we are on a crest of a wave because they like people who get people out of hospital quickly, so we are certainly very lucky with that, but they are very concerned about activity based funding

Evidence from the peer review literature and activity and workforce data were sources of information physiotherapists and physiotherapy managers considered when making decisions about physiotherapy service provision. Drawing upon the available evidence base was noted to be particularly important when making decisions about resource allocation when the demands exceeded available resources. Data, while limited in many areas, was used by both physiotherapists and their managers to manage rostering and workload allocation, for activity reporting, and in submissions for funding of new services. Research evidence was used to inform decisions about service provision, yet was considered "a two edged sword" by some as the physiotherapy research base does not yet reflect all areas of practice. Lack of time limited greater participation in research to develop a local body of evidence.

D1: ... where the resources are best spent, we've very much put that on current practice and research.

A6: ... provide them with stats with what we're doing, what we're able to do, not able to do, give them suggestions of how we can streamline services, save them money, and I guess let them know what happens when we are short staffed.

A2: ... we haven't done any sort of study on that and I think that they're hand in hand, we can only grow the service if we've got the evidence of best practice that we can then put to people who have money and purses and strings, but we will never be able to, it would take a real reshuffle of how we do our work as to part timers to actually see how we could incorporate that. Certainly we read widely, but we won't be able to show people evidence. 
D1: ... evidence based practice, is a double edged sword in my opinion, because a lot of things we do don't have evidence.

In both regional and rural sites, perception, knowledge, and awareness of the role of physiotherapy appeared to have both positive and negative influences on physiotherapy services. This was particularly important where services or teams have managers and leaders who are not physiotherapists. Trust was reported as a key factor by one participant, with frustration and missed opportunity evident in other responses. These contribute to the physiotherapist perceptions of organisational support; a key workforce retention factor. ${ }^{24}$

A5: Management makes a huge difference. I have a really supportive manager and she trusts me implicitly ... let's me get on and do the job; understands my clinical needs.

D1: I know there are places where we've not been positively viewed to start with and then as we've gone on and just worked in a team and whatever, it's been much more seen as a positive thing and then people have been advocating for more of it [physiotherapy].

A5: We had a terrible one [manager] when she was away ... he was here for a year ... a lot of people resigned. It was devastating what he did in that time.

\section{Directives or Choice}

The level of autonomy emerged as a differentiating factor between services, most notably between public and private sector providers. Autonomy and scope of SLDM varied with the stakeholder's sector and position. Private sector physiotherapists revealed higher levels of autonomy in decision making about service provision. In contrast, public sector physiotherapists had less autonomy in determining service provision. This was most evident in larger facilities where physiotherapy led decisionmaking was constrained by organisational priorities, service directives, and decisions made at higher levels of the health system. Frustration was evident in the responses of some public sector physiotherapists when service directives were given without consultation or consideration of physiotherapy capacity to meet current and new service requirements. Private physiotherapists reported providing services that matched their areas of interest and expertise and were financially viable. Public physiotherapist responses revealed a greater sense of obligation to meet the needs of the broader community and organisational priorities. Tensions and conflict were evident in public sector participant responses when resource constraints limited the range of services provided.

A5: Well I don't have control over our budget, and to be honest I don't even know if our community health manager does.

A3: In terms of demand, sometimes services are just created, sometimes decisions are made here without consultation on where the allied health services will be provided. They might re-jig rehab and decide they are opening four more beds but there's no thought whatsoever into whether physio or OT or anyone is capable of taking on another four beds. Decisions like that will be made and still people aren't consulted particularly well.

B1: I don't believe I have to provide treatment to people who can't afford to pay me for it. I like doing what I do and I like doing it but not in a hospital setting. Is it choice? I still see and do things and stay later than I want to stay so that's not choice in that way, but it gives me pleasure in making my practice what I want it to be.

Private practitioners described two attributes that constrained their SLDM. One was the compelling aspect of financial viability; if you don't work there is no income. This was especially relevant for sole practitioners and the long term sustainability of their business. While financial viability was an important SLDM consideration for physiotherapists in private practice, making money was not always the biggest incentive. The second attribute is self-reliance; critical factors include a combination of skill and expertise and physical capabilities.

B1: You can't afford to run at a loss but making money has never been my biggest incentive.

B4: I need to make money to cover my costs, but money's never been a driving force in me working, and my attitude is, I want to do a good job and I want to help these people help themselves. 
B1: I'm more aware of my physical limitations and I'm much more likely to say now, than I was 10 years ago, just don't book any more.

Distance from regional, state, and national decision makers was an emerging theme across public sector services. Distance was viewed as either geographical distance from regional decision makers or hierarchical distance within organisations or facilities. Despite devolution of decision making forming a cornerstone of recent organisational restructures, there remained a sense that decisions influencing service provision occurred centrally at a distance from the service providers, often with limited transparency. Recent changes at state level were also noted to limit opportunity for participation and input by rural health professionals.

D4: [A previous restructure] saw the old districts collapsed into areas ... [Their] responsiveness to needs out here is pretty low. But there was an Allied Health Advisor, I don't know if he ever got out here, I've never met him.

A1: The interesting thing about here though is who decided what got cut and how much? There's not necessarily a lot of logic in that, it was just bottom line, it was just dollars and the positions were cut if they were not filled by permanent employees. It wasn't about services, it was about money.

D1: Yes it is hard to tease out, because does someone sitting in an office somewhere say we'll have some physio but we won't have some radiography, I don't know.

A2: [Factors influencing decisions] tend to be fairly external ... they tend to be from positions that aren't potentially at the coal face.

D2: We've actually, over years, centralised people to rural locations and that we will outreach to a remote location should they need to.

A5: Everything is centralised to regional and metropolitan; they are in their bed block constantly - they're constantly being harassed on their pages and over the phone system; 'We need beds; we need beds'.

A8: And they're sort of hamstrung by policies that come from [the capital city] and so we don't even have a rural health task force anymore, that's been stopped ... rural people have trouble getting on those committees and also their small voice. Whereas before, at least we had some voice.

\section{Voice at the Table}

The importance of having a "voice at the table" where decisions are made was noted by many physiotherapists. Such comments were provided in response to the question: "Who else contributes to, or influences, the decisions about physiotherapy service provision within your facility/service and how do they do so?" The level of participation in facility level decision making varied between sites and was valued where physiotherapy representation occurred. One physiotherapy decision maker pragmatically recognised that they while they "lacked the clout" at a facility level, they were able to determine where they allocated physiotherapy resources.

A3: Yes, representation, making sure we're there, sitting on committees and making physio a relevant service to be represented on that.

A6: It's great. And to have that knowledge of what's happening within the hospital, before that flow down wasn't happening. So we'd be the last to know that there's a redevelopment or there's a change in some other service or plan or directive of the hospital.

D1: I don't think we have enough clout to say which services are and aren't provided or a priority of the hospital. We have input into what we provide in that but because we're always trying to work out where we're getting our best bang for our buck and where the resources are best spent. But it's not us having the say over the development of that service; it's us going with it.

\section{Connection to Community: Visibility and Accountability}

Health professionals in smaller rural settings reported an increased community visibility, which was regarded both as a positive and negative factor in terms of retention. Visibility within a rural community was at times accompanied by an increased sense of 
accountability, both of which can impact on decisions about physiotherapy service provision.

D4: It's a really interesting dynamic but I think it makes you more responsive to what the needs are of the community. In terms of retention, it's a double-edged sword. So you hear people who are happy in their work ... about how nice it is to be connected in the community and the patients know you and you're valued and they say hello to you at Woolies. As you see people become either tired or disillusioned, the language around the same issue changes dramatically. So I've got no privacy, even in the supermarket people want to talk to me - so it's exactly the same thing, but how it's perceived and described is vastly different. So I guess it does impact on how you provide your service. I don't think it's a constant. So if everything's good - like if everything's going well around somebody, it tends to be viewed as a positive. If other things in their work environment, well particularly their work environment aren't acting the way they'd like it, then all those things become negatives.

A7: [In a smaller community] you see much more directly when you are there what happens and then when you're not there what happens, and for me a much higher sense of accountability.

D4: In terms of service delivery I think it does make it much harder to not respond - I think it puts pressure on people. How much pressure ..., I don't think it's enormous. But I think people do think a little bit about it. If I shut the door at one minute to four, and somebody knocks on the door and I say, 'No, I'm closed'. It's very different because you know you're going to see them.

\section{Rurality and Size}

Consistent with existing rural physiotherapy literature, issues of recruitment and retention of staff, workload and the generalist requirements of rural practice were noted. Participants in this study provided insights into how these factors influenced decision making about rural physiotherapy service provision. SLDM factors identified also included reporting to managers with qualification other than physiotherapy, the impact of service decisions being made in larger regional centres, and the importance of multidisciplinary team colleagues, yet being a lone voice for physiotherapy. The capacity of a rural community to support and sustain private physiotherapy providers was also noted. Recent years had seen the establishment of private practices in towns with populations of around 10,000 to 15,000. The limited capacity of many smaller or more remote communities to sustain a private practice increased reliance on public physiotherapy service providers.

D4: The fact that the health service does operate a pretty comprehensive service, there's not a lot room left in the market. If we were to reduce our services, yes it'd expand the market for private providers, but whether or not we'd get any private providers or have a community that could afford to access the private providers I don't think is the real question. I don't think that the population could actually support a significant private practice. I think that's one of the big drivers around why the system's the way it is.

A8: I'm the only allied health professional really, as a therapist based [here].So I'm probably, as an allied health professional, more isolated than more remote areas. There have been times this year that l've been the only one [physiotherapist] in thirty thousand square kilometres. It's difficult when you're by yourself and have to do all the bookwork. Its management and I'm on a lot of committees for the hospital and you've got to prepare for the bureaucratic nightmares of the EQuIP [Health Service Accreditation].

\section{A Conceptual Map of Factors Influencing Rural Physiotherapy Service Level Decision Making}

The results of this study informed the development of a conceptual map of factors influencing rural physiotherapy SLDM (Figure 1). Building on a diagrammatic representation of multilevel factors influencing the provision of physiotherapy services, participant perspectives suggest that decision making varies with sector, size, and rurality. As with factors influencing actual service provision, factors influencing SLDM occurred at macro, meso, and micro levels of the health system. The relative impact of SLDM factors varied. National and state policy and funding, for instance, influenced public sector SLDM more so than that of private sector physiotherapists. While capacity and capability were common across sites and sectors, the size of the service strongly influenced staffing levels, skill mix, and the ability to recruit and retain physiotherapists. Recruitment to smaller services requiring generalist physiotherapists was often challenging,and where prolonged vacancies occurred, people in the community had limited or no access to local physiotherapy services. Larger regional facilities reported challenges in recruiting to senior positions and vacancies resulted in a loss of specialised physiotherapy services for the local community and the surrounding region. SLDM was more reactive and constrained where there were multiple or prolonged vacancies. Rural services noted a stronger connection to the local community, accompanied by a sense of increased visibility and accountability while also highlighting the challenge of being distant from decision making. Decision making at the physiotherapy service (micro) level was

(c) The Internet Journal of Allied Health Sciences and Practice, 2015 
informed and influenced by decisions made at regional and facility (meso) level, and national and state (macro) level. Adopting a systems approach to explore rural physiotherapy SLDM enabled consideration of the broader health system. Conceptualising the influencing factors in this manner may assist rural physiotherapists and other decision makers with future SLDM.

Figure 1. Conceptual Map of Rural Physiotherapy Service Level Decision Making

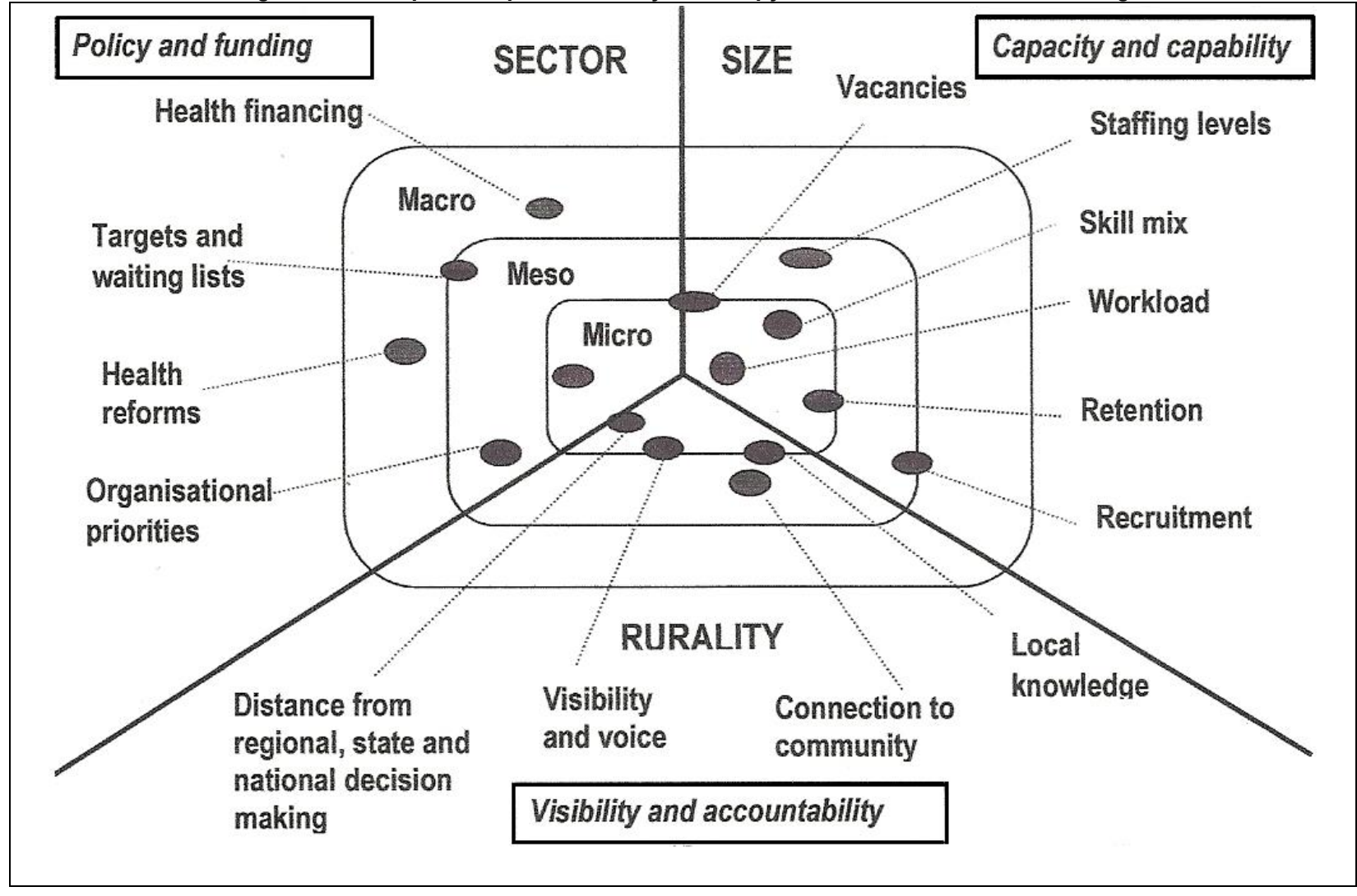

DISCUSSION

The findings of this study will be familiar to rural health professionals and to decision makers with responsibilities for rural health service provision. By seeking participant perspectives on decision making about the provision of physiotherapy services across a range of rural communities, this study sought to give voice to what is discussed at a local level. The findings affirm what is known, while adding insight into the detail at a local service level and discerning differences in physiotherapy services in the communities of this study. Obtaining perspectives of a range of stakeholders such as physiotherapists, their multidisciplinary colleagues, consumers, managers, and other decision makers provided a richer view of SLDM than a physiotherapy only perspective would provide.

Decision making about rural physiotherapy service provision varied with the service size, location and sector. The conceptual map provided in Figure 1 summarises clusters of issues under broad headings such as capacity and capability, policy and funding, and visibility and accountability. Physiotherapy services were more influenced or more sensitive to some factors more than others. For example, participants in smaller rural communities were influenced by a stronger connection to the community and a sense of increased accountability when making decisions about service provision. Some factors, such as physiotherapy workforce availability and capacity, were common across services, sites and sectors. Whether public or private, small or large, rural or regional, the availability of specific physiotherapy expertise and experience relevant to a service underpinned decisions about services provided. However, service size determined the nature of physiotherapy expertise required and the degree of vulnerability to prolonged vacancies.

Challenging recruitment and retention of physiotherapists in rural areas is well described. However, smaller services with less critical mass were more vulnerable to workforce or skills shortages. Smaller services included smaller rural physiotherapy services, small private practices, or specialised services in regional centres provided by a single physiotherapist. Vacancies increased workload of remaining physiotherapists and at times posed a risk to the sustainability of the service. Prolonged 
vacancies created services gaps and a loss of funding for positions was also reported. Consistent with the rural physiotherapy literature, participants in this study identified workforce supply was a critical factor underpinning service delivery and a key consideration when deciding what services to provide.

Service or facility size largely determined the type of physiotherapy service provided and the expertise required. Larger regional health facilities required a mix of generalist and specialised expertise to meet the service requirements. Generalist services and expertise were more common in smaller physiotherapy services and musculoskeletal services and skill requirements common in the private practices of this study. Within public facilities, the proportion of physiotherapy services provided to inpatient physiotherapy increased with facility and community size as did the availability of specialised services. Conversely, the smaller the community, the greater proportion of time was allocated to outpatient physiotherapy service provision. This was important in smaller rural communities that were unable to sustain private physiotherapy services.

Size also influenced the level of discretion in SLDM. Larger regional services had more layers of decision makers (such as department manager, allied health manager, operational manager, and general manager) compared to smaller facilities. Physiotherapists in smaller facilities had fewer organisational reporting layers, some reporting directly to the health service manager or through a community manager to the health service manager. Physiotherapists in smaller facilities, as with principals of private practices, reported greater independence in making decisions about physiotherapy service provision.

The prerogative to choose what services to provide emerged as a distinguishing factor between public and private sector services. The principals of private practices reported the choice of services provided was theirs to make. This contrasted with the public sector, particularly the larger services, where alignment to organisational or funding priorities influenced decisions about physiotherapy service provision. An early assumption of the researchers was that SLDM would not be the sole prerogative of physiotherapists. This assumption appears valid in public sector services, more so in larger organisations, but the principals of private physiotherapy practices do hold sole SLDM decision making prerogative. While the latter is perhaps not an unexpected finding, it was not an expressed assumption at the outset of the study. Influencing groups or contributors to public sector physiotherapy SLDM included clinical colleagues (nursing, medical and allied health), managers (facility, regional and state), referrers (doctors, other service providers), the community, and the media.

State and national (macro) level policy and funding decisions were perceived to be more influential within public sector physiotherapy services than private sector services. National health reforms and changes to health financing generated organisational priorities at state, regional, and facility level that influenced decision making about local physiotherapy service provision. At a national level, key influencing factors included the introduction of activity based funding, formation of local health boards, and establishment of national performance targets. New policy directions at a state government level then framed decision making at a regional and facility (meso) level. Fiscal austerity measures and targeted service funding designed to achieve specific patient outcomes were two examples. In larger regional facilities, service targets and organisational priorities, such as optimising length of stay and revenue, then influenced service (micro) level decisions about physiotherapy service provision. In smaller rural services, local knowledge and consideration of community need were more influential SLDM factors.

SLDM in smaller rural communities was further influenced by a sense of increased accountability and obligation that comes with being part of the community. Community need, local demand and practitioner defined priorities were more common decision drivers in smaller rural facilities. Emerging from participant comments was the notion of a SLDM continuum based on community connection and distance from regional, state, and national decision makers. Decision making informing service provision or service boundaries often occurred higher up in organisations, at some distance from the face of patient-provider interaction. Physiotherapy participants reported professional conflict when access to services was limited by criteria (such as age or geography) specified by macro level decision makers who had limited connection to the local community. One physiotherapist, who lived and worked in the same community, exemplified this conflict when stating that they were "... much more uncomfortable with drawing the line that says you are eligible and you are not due to geography or the year you were born or whatever" [A2:].

In summary, rural physiotherapy SLDM varied with sector, size and rurality. Private physiotherapy practitioners have the most discretion in the services they provide. Public sector physiotherapy services are constrained by government policy and organisational imperatives. The smaller the service, the fewer levels of bureaucracy and less acute inpatient care which allowed greater flexibility at the service delivery level. Ultimately, however, services cannot be provided if the service is unable to attract clinicians with the requisite skills. Recruitment to regional, rural, and remote communities remains challenging and attracting physiotherapists to smaller rural communities or physiotherapists with specialised expertise required in regional services is critical to service sustainability and a key consideration in SLDM.

(c) The Internet Journal of Allied Health Sciences and Practice, 2015 


\section{Limitations}

This study was undertaken in one region of Australia that had a mixture of regional, rural, and remote centres. Results may not be applicable to other areas with a different mixture of centres such as more remote locations and less regional centres. Although this study asked physiotherapists to identify decision making stakeholders, not all stakeholders were involved as participants. This may have biased results; for example, state health department decision makers were not involved in this study and yet their decisions will influence the physiotherapy service provision in rural and regional areas. This study only investigated decision making related to rural physiotherapy services provision decision making. The results may not be applicable to other health disciplines and there may be different factors and interactions in setting where there are other service delivery models.

\section{CONCLUSION}

Multiple interrelated factors informed SLDM about rural physiotherapy service provision. System level influences from macro and meso level decision makers provide the framework within which micro level physiotherapy SLDM occurs. Service sector, size, and rurality then further qualify local service options and influence SLDM. Understanding context and diversity is important to understanding local implementation of health system decisions and gaining insight into the variation in service provision between sites. The findings in this study are consistent with existing rural physiotherapy literature and reinforce the importance of physiotherapy workforce availability to service sustainability. This study adds to the rural physiotherapy literature by providing greater insight into decisions informing physiotherapy service provision. Exploring decision making about rural physiotherapy service provision within a systems-theory case study heuristic enabled consideration of the impact of contextual factors such as service location, size, and sector. Considering participant responses within this heuristic framework assisted in discerning commonalities and differences between sites and services.

Decisions made at macro and meso levels directly influence decisions at a local service level. At times, decisions about service provision resulted in duplication or gaps in services to people in the communities of this study. This was evident when new services were funded with narrow service criteria which limited eligibility. Decisions about service provision need to take into consideration the current availability of services, the context of each location, and the skill mix required. Devolved decision making may then optimise service delivery to meet local community needs whilst reflecting state and national policy and directives. An inclusive approach to devolved SLDM may produce better solutions for local service provision. Local decision making is further enhanced by having the right "voices at the table," knowledgeable about their role and "connected to the community."

\section{REFERENCES}

1. Larson A. Rural Health's demographic destiny. Rural and Remote Health. 2006;6(551).

2. Sheppard LA. Work practices of rural and remote physiotherapists. Aust J Rural Health. 2001 Apr; 9(2):84-90. [PMID: 11259962]

3. Williams E, D'Amore W, McMeeken J. Physiotherapy in rural and regional Australia. Aust J Rural Health. 2007; 15(6):380-6.

4. Council of Australian Governments. National Health Reform Agreement. Retrieved February 17, 2015 from http://www.publichospitalfunding.gov.au/national-health-reform/agreement.

5. Department of Health and Aging. Programs and Initiatives: Health workforce. 2014. Retrieved 1 February 2014, from http://www.health.gov.au/internet/main/publishing.nsf/content/work-pr-alli

6. Services for Australian Rural and Remote Allied Health. Nursing \& Allied Health Scholarship and Support Scheme. 2013. Retrieved 25 January 2014, from http://www.sarrah.org.au

7. Struber J. Physiotherapy in Australia: Where to now? The Internet Journal of Allied Health Sciences and Practice. 2003 July; $1(2)$.

8. Department of Health and Aging. Chronic Disease Management - Individual Allied Health Services under Medicare. 2013. Retrieved 25 January, 2014, from http://www.health.gov.au/internet/main/publishing.nsf/Content/health-medicarehealth_pro-gp-pdf-allied-cnt.htm

9. Australian Physiotherapy Association. The Factors affecting the supply of health services and medical professionals in rural areas: Submission to Senate Community Affairs Committee. 2011. Melbourne. Australian Physiotherapy Association.

10. Sibbald SL, Singer PA, Upshur R, Martin DK. Priority setting: what constitutes success? A conceptual framework for successful priority setting. BMC Health Services Research. 2009;9:43. Epub 2009 March 5. [PMID 19265518]

11. Chapman G, Sonneberg F, editors. Decision making in Health Care: Theory, psychology and applications. Cambridge: Cambridge University Press; 2000.

12. Eagar K, Garrett P, Lin V. Health Planning: Australian Perspectives. Crows Nest: Allen \& Unwin; 2001

13. Edwards I, Jones M, Carr J, Braunack-Mayer A, Jensen GM. Clinical reasoning strategies in physical therapy. Phys Ther. 2004 Apr; 84(4):312-30; discussion 31-5. [PMID: 15049726]

(c) The Internet Journal of Allied Health Sciences and Practice, 2015 
14. Edwards I, Braunack-Mayer A, Jones M. Ethical reasoning as a clinical-reasoning strategy in physiotherapy. Physiotherapy. 2005;91(4):229-36.

15. Edwards I, Richardson B. Clinical reasoning and population health: decision making for an emerging paradigm of health care. Physiother. 2008 May-Jun; 24(3):183-93. [PMID: 18569855]

16. Finch $E$, Geddes EL, Larin $H$. Ethically-based clinical decision making in physical therapy: process and issues. Physiother. 2005 Jul-Sep; 21(3):147-62. [PMID: 16389696]

17. Grimmer-Somers K. Incorporating research evidence into clinical practice decisions. Physiother Res Int. 2007 Jun;12(2):558. [PMID: 17536643]

18. Smart K, Doody C. The clinical reasoning of pain by experienced musculoskeletal physiotherapists. Manual Therapy. 2007 Feb; 12(1):40-9. [PMID: 16781185]

19. Smith M, Higgs J, Ellis E. Physiotherapy decision making. Aust J Physiother. 2007;53(4):261-7. [PMID: 2009748011.]

20. Smith M, Higgs J, Ellis E. Characteristics and processes of physiotherapy clinical decision making: a study of acute care cardiorespiratory physiotherapy. Physiother Res Int. 2008 Dec;13(4):209-22. [PMID: 18985624]

21. Smith $\mathrm{M}$, Higgs J, Ellis $\mathrm{E}$. Effect of experience on clinical decision making by cardiorespiratory physiotherapists in acute care settings. Physiother. 2010 Feb;26(2):89-99. [PMID: 20067358]

22. Miller-Mifflin TM, Bzdell M. Development of a physiotherapy prioritization tool in the Baffin Region of Nunavut: a remote, under-serviced area in the Canadian Arctic. Rural Remote Health. 2010;10(2):1466. [PMID: 20572750]

23. Bourke L, Humphreys JS, Wakerman J, Taylor J. Understanding rural and remote health: A framework for analysis in Australia. Health \& Place. 2012;18(3):496-503.

24. Campbell N, McAllister L, Eley D. The influence of motivation in recruitment and retention of rural and remote allied health professionals: a literature review. Rural Remote Health. 2012 June;12:1900. Epub 2012. 